\title{
Role of Convection Flow on the Pattern Formation in the Drying Process of Colloidal Suspension *
}

\author{
MA Wen-Jie(马文杰), WANG Yu-Ren(王育人)**, LAN Ding(蓝鼎) \\ National Microgravity Laboratory, Institute of Mechanics, Chinese Academy of Sciences, Beijing 100080
}

(Received 10 December 2007)

\begin{abstract}
We study the macroscopic drying patterns of aqueous suspensions of colloidal silica spheres. It was found that convection strength can influence pattern formation. Uniformed films are obtained at weaker convection strength. In addition, we make clear that it is not reasonable to discuss individually the effect of temperature and humidity on the colloid self-assembly. The physical mechanism is that these factors have relationship with the evaporation rate, which can affect the convection strength.
\end{abstract}

PACS: 47.20.Ma, 47.54.-r, 68.37. HK

Drying processes, such as the soil drying process and the paint drying process, play an important role in industry and our daily life. Generally speaking, drying patterns in these processes are dominated by the dissipation of free energy and the self-organization of coarsely or finely granular matter in open systems. Recently, well-controlled drying process of colloidal particles was also be used to synthesize the functional material such as photonic crystal. To understand the formation mechanism of the dissipative structures, great attention has been devoted to the structural pattern formation in the drying process of colloidal suspension. The process was dominated by many factors involving convectional flow, evaporation and interaction of colloidal particles, which make it difficult to have a comprehensive understanding of the phenomenon. An obvious feature of the drying pattern of colloidal suspension is the appearance of similar macroscopic broad ring patterns irrespective of the kind of the suspensions and solutions. ${ }^{[1-6]} \mathrm{A}$ series of sedimentation and drying dissipative patterns for aqueous suspensions of colloidal silica spheres were studied and clarified that the broad-ring patterns were formed unexpectedly fast in the suspension state. ${ }^{[7]}$ The drying process was considered to be a selforganization process, which was used in fabrication of various nanostructured materials and devices. ${ }^{[8]}$ Since a large-scale nanostructured film with a flat and uniform surface is anticipated for applications in photonic crystal engineering, it is necessary to avoid broad-ring formation.

Although the previous research ${ }^{[9]}$ studied phenomenally on the role of capillary force and convectional flow in the dissipative pattern formation, the physical picture is not clear due to many factors governing the phenomena. Many factors can affect the self-assembling process of colloidal particles, such as temperature, relative humidity, sphere diameter, col- loidal concentration and even the titling angle of the substrate. McLachlan et al. ${ }^{[10]}$ reported that the colloidal concentration can be used to control the domain size. Ye et al. ${ }^{[11]}$ suggested that the integrality of colloidal self-assembly strongly depends on the evaporation temperature. Chung et al. ${ }^{[12]}$ have fabricated high-quality colloidal crystal by increasing the system humidity. Although these studies reflected the effect of individual factor on the pattern formation, further work should be carried out to clarify the relationship between these factors and to give a clear picture for the pattern formation mechanism in physics.

In terms of fluid mechanics, the movement of nanometre-sized colloidal particles is dominated by sedimentation and convection in the suspension. Sedimentation itself cannot affect the radial distribution of colloidal particles and influence the formation of various patterns if there were not any kind of convection in colloidal suspension. Therefore, we should focus on the study of convection effect on the dissipative structure formation. We noticed that convection strength can be evaluated qualitatively by the evaporation rate in the drying process, which is a function of temperature, humidity and pressure. Routh and Russel proposed a Peclet number $P e$, defined as

$$
P e=H E / D_{0},
$$

where $H$ is the initial thickness of the film, $E$ the rate of evaporation, $D_{0}$ the diffusion coefficient of the colloids. ${ }^{[13]}$ The Peclet number is used to denote relative proportion of convection and diffusion. From the above equation, it is easily to be understood that the convection strength has a proportional variation with the evaporation rate. In this Letter, we report our study on the influence of convection strength by varying the evaporation rate. Different from the previous studies, we slightly change the geometry of the drying

\footnotetext{
* Supported by the Knowledge Innovation Project of Chinese Academy of Sciences under Grant No KJCX2-SW-L05.

**Email: wangyr@imech.ac.cn

(c) 2008 Chinese Physical Society and IOP Publishing Ltd
} 
cell to uniform the radial gradient of the temperature in the cell. The present study can verify the role of capillary convection on the pattern formation.

The silica spheres were dispersed into the mixture of ethanol and deionized water with the assistance of an ultrasound bath forming a $1.5 \mathrm{wt} \%$ suspension. The diameter of silica colloidal particles is $300 \mathrm{~nm}$, and the relative deviation in size is less than $5 \%$. The glass substrates were rinsed with deionized water and dried under a flow of nitrogen gas. The glass substrate was placed horizontally on the bottom of the Teflon container with a lid. For each sample, a circular silica glue mat with $1.3 \mathrm{~cm}$ inner diameter was set onto a glass substrate, and $180 \mu \mathrm{L}$ colloidal suspension was dropped onto the glass substrate. The Teflon cells were placed into a temperature-humidity-constant incubator. The drying process was conducted at temperature of $50^{\circ} \mathrm{C}$ with humidity of $35 \%$.

Macroscopic patterns were observed with a canon digital camera. The microstructures of colloidal crystal films were observed with a scanning electron microscope. Weight loses of the films were measured by a balance (precision of 0.001, measured at one hour time-interval). Figure 1 shows the optical photographs of dried films. The colloidal suspensions for different samples were dried in the cylindrical containers covering with lids of different holes in the central part (as shown schematically in Fig. 2(b)). The holes diameters for samples A, B, C, and D are $10 \mathrm{~mm}$, $7 \mathrm{~mm}, 5 \mathrm{~mm}$ and $2 \mathrm{~mm}$, respectively. The thicknesses of the films are almost equal to $9 \mu \mathrm{m}$ and consist of 30 layers of colloidal spheres. Figures $1(\mathrm{a})$ and $1(\mathrm{~b})$ show that obvious broad-ring patterns appear, which are consistent with the previous report. ${ }^{[14]}$ The appearance of ring pattern indicates that the thickness of the dried film is not uniform and has an oscillatory variation in the radial direction. Another feature of such a kind of films is the appearance of void in the central part of the films. This is due to the strong segregation of the colloidal particles towards the periphery region of the container in the drying process. It should be noted that the segregation was driven by the convective flow in the suspension. However, with further decrease of the hole diameter, Figs. 1(c) and 1(d) show that the broad-ring patterns could not be observed again. Instead, the relatively uniform films are obtained. The spoke-like striations in Figs. 1(c) and $1(d)$ are caused by the cracks. Although the cracks cannot be observed clearly in Figs. 1(a) and 1(b), they do exist and could be observed in the SEM low magnification images for samples $\mathrm{A}$ and $\mathrm{B}$. Moreover, the voids occurring for samples $\mathrm{A}$ and $\mathrm{B}$ are covered by the thin layers of colloidal particles for samples $\mathrm{C}$ and D. This is because the more homogeneous distributions of colloidal particles are obtained in samples $\mathrm{C}$ and D.
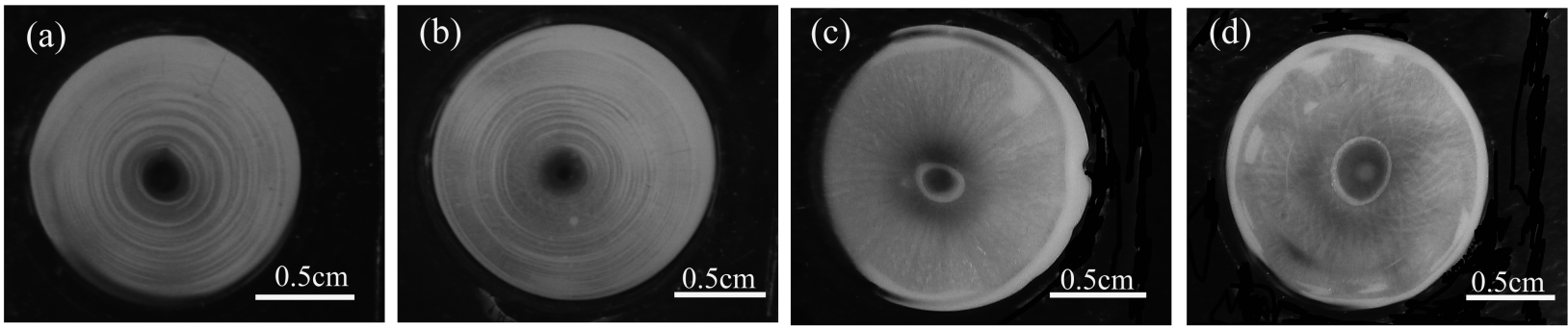

Fig. 1. Drying patterns formed for colloidal silica spheres at different evaporation rates with lid holes of different diameters: (a) $10 \mathrm{~cm}$, (b) $7 \mathrm{~cm}$, (c) $5 \mathrm{~cm}$, (d) of $2 \mathrm{~cm}$.

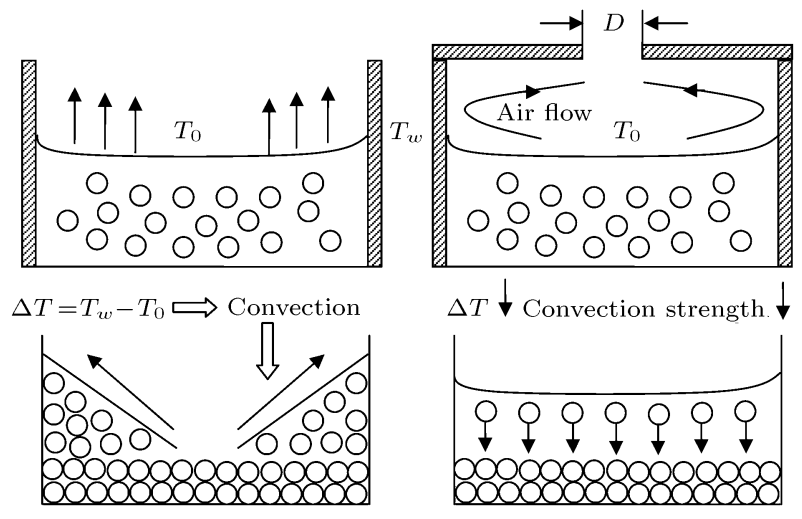

Fig. 2. A schematic of experimental setup under different convection conditions with and without lid: (a) drying without lid, (b) drying with lid.
To describe the mechanism of pattern formation for our experiments in convenience, we depict the schematic of drying cells in Fig. 2. As shown in Fig. 2(a), for an open container, the water vapour directly evaporates from the surface of colloidal suspension. The segregation of colloidal particles is mainly driven by the surface tension-induced convection (the so-called Marangoni convection) for a shallow cell. It is well known that the strength of Marangoni convection depends on the temperature gradient along the drying cell surface, while the gravity-induced buoyancy convection can be neglected due to the space limitation in vertical direction. Figure 2(a) shows that broad-ring pattern forms for drying without lid, which is mainly caused by the Marangoni convection. The 
rate of evaporation at the edge is greater than that at the centre. To compensate for water loss at the edge, the capillary flow occurs from the centre to the edge, enabling colloidal particles to flow and accumulate near the round edge. ${ }^{[15]}$ Contrary to the above discussed case, we put lids with different diameters holes on the drying cells where the drying patterns are shown in Fig. 1. As discussed in the last paragraph, a pattern transition from broad ring to uniform film is found in the experiments with the decreasing hole diameter. Figure 2(b) schematically shows the drying cell. In our experiment, since the liquid layer is very thin, the gravitational convection can be left out. Compared with the drying process in Fig. 2(a), the vapour air has to flow from cell rim region to central part. This radial vapour air flow will uniform the temperature distribution along the water surface in the cell. It is well known that the uniformity of temperature will make the Marangoni convection strength descend, which results in the less amount of colloid congregation at the edge. Therefore, a uniform and flat film can be obtained, which explains the phenomena shown in Figs. 1(c) and 1(d).

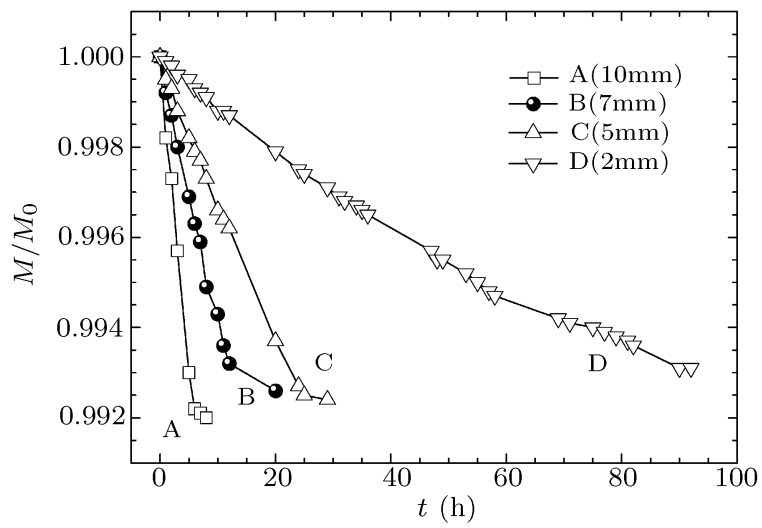

Fig. 3. Relative weight of colloidal suspensions as a function of the normalized time $t$.

Figure 3 shows $M / M_{0}$ as a function of the drying time. We measure the weight changes under various evaporation conditions. At the beginning of the drying, the relative weight decreases linearly with time for each sample, which indicates that the evaporation rate is basically constant. From the results, we can see that with decreases of the inner diameter of the hole, the evaporation rate decreases. The evaporation rates of samples A, B, C, D are 0.0621, 0.0150, 0.0072, $0.0017 \mathrm{~g} / \mathrm{h}$, respectively. According to Eq. (1), with the decrease of the evaporation rate, the convection strength decreases, therefore more uniformed films re observed in Figs. 1(c) and 1(d).

Temperature and humidity can influence the selfassembly of colloidal particles. The essential reason is the relationship between temperature, humidity and evaporation rate. In several studies of evaporating liquid layers, ${ }^{[16-18]}$ the mass flux $J$ is assumed to obey the Hertz-Knudsen equation, derived from the kinetic theory of gas. ${ }^{19]}$ The non-equilibrium law is derived for a flat interface along which the equality of liquid and vapour temperature is assumed (the vapour is further assumed to be a perfect gas), and reads

$$
J=\beta \sqrt{M /(2 \pi R T)}\left[P_{s}(T)-P_{0}(T)\right],
$$

where $\beta$ is the accommodation coefficient, $M$ is the molecular weight of vapour, $P_{s}(T)$ is the saturation pressure at surface temperature $T, P_{0}(T)$ is the vapour pressure just beyond the interface, $P_{0}(T)=P_{s}(T) \phi$, $\phi$ is the relative humidity. Obviously, the changes of temperature and relative humidity lead to change of the evaporation rate finally. For our system, temperature is fixed up, and the changes of evaporation rate come from the changes of $\phi$. According to the results of evaporation rate stated above, the humidity in the cells are estimated to be $56 \%, 81 \%, 94 \%, 98 \%$, respectively. It should be noted that the humidity values declared herein refer to the humidities near the suspension surface in the containers, while the humidity of $35 \%$ declared in the experimental part refers to the environmental humidity. According to Eqs. (1) and (2), we know that both temperature and humidity can influence the evaporation rate, which has effect on the strength of convection flow. Therefore, convection strength is a more essential factor and should be emphasized when we discuss the self assembling process of colloidal particles, instead of discussion on the individual influence from temperature or humidity.

Crack is affected by the inner stress in the film. Different patterns formed in the drying process will produce different cracks. In addition, large domain size is critical for producing high quality template for subsequent infiltration and formation of ordered nanostructured materials. Crack structures ${ }^{[10]}$ were observed at different evaporation rates. Figure 4 shows the spoke-like cracks formed in our experiments. The cracks nucleate at or close to the rim, and then grow in radial directions. It can be seen that the numbers of cracks and the domain sizes are not the same for the specimens. An increase of the domain size via the decrease of the evaporation rate and convection strength is observed. For samples $\mathrm{A}$ and $\mathrm{B}$, the domain sizes are much smaller than those of samples $\mathrm{C}$ and $\mathrm{D}$. As can be seen from Figs. 4(a) and 4(b), for samples A and B, the domain sizes are $150 \mu \mathrm{m} \times 100 \mu \mathrm{m}$ and $400 \mu \mathrm{m} \times 100 \mu \mathrm{m}$, respectively. For samples $\mathrm{C}$ and $\mathrm{D}$, the evaporation rates are quite slow, which make the drying shrinkage stress reduce. The domain sizes are approximately $500 \mu \mathrm{m} \times 150 \mu \mathrm{m}$ and $600 \mu \mathrm{m} \times 200 \mu \mathrm{m}$, respectively, as shown in Figs. 4(c) and 4(d). These observations con- 
clude that large domain sizes can be obtained at slower evaporation rate and at lower convection strength.

Figure 5 shows the high magnification SEM images of silica colloidal crystals at different evaporation rates for different convection strengths. As can be seen from Figs. 5(a) and 5(b), samples A and B contain more disordered areas and vacancies. Samples $\mathrm{C}$ and $\mathrm{D}$ fabricated at a lower evaporation rate have fewer defects (Fig. 5(c) and 5(d)). These results indicate that the crystalline quality strongly depends on the evaporation rate and convection strength. Fast solvent evaporation and high convection strength induce colloidal spheres packed in a disordered manner since they have no enough time to move into ordered equilibrium positions.
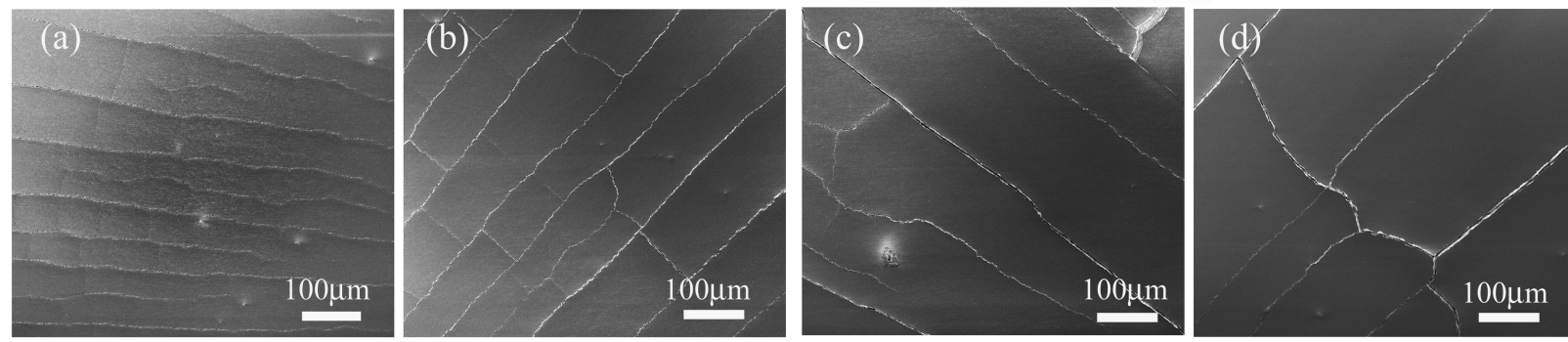

Fig. 4. Low magnification SEM showing a dramatic increase in macroscopic domain size with decrease of the rate of evaporation: (a) sample A, (b) sample B, (c) sample C, (d) sample D.
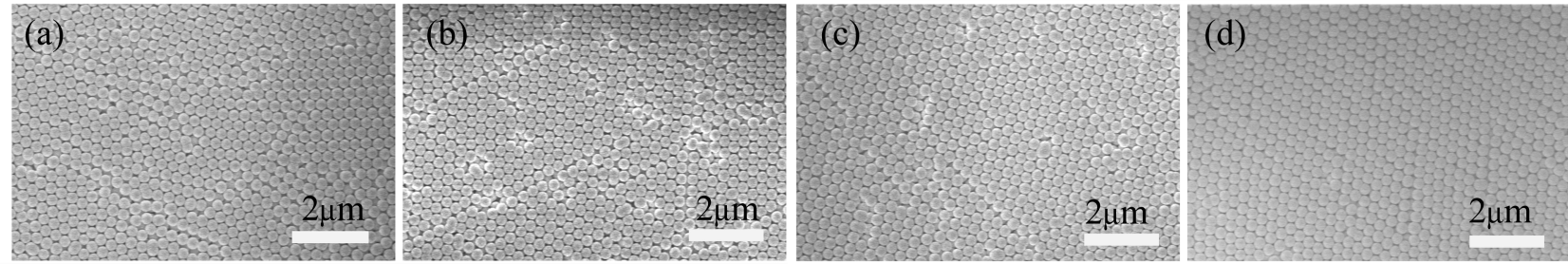

Fig. 5. High magnification SEM showing crystal quality at different evaporation rates: (a) sample A, (b) sample $\mathrm{B}$, (c) sample C, (d) sample D.

In summary, our experiments show that drying pattern formation of colloidal spheres can be affected by the convection strength. Convection strength is a more essential factor and should be emphasized when we discuss the self-assembling process of colloidal particles, instead of discussion on the individual influence from temperature or humidity. Homogeneous films with smaller circular void can be produced at weaker convection strength.

\section{References}

[1] Okubo T, Okuda S, Kimura H 2002 Colloid Polymer Sci. 280454

[2] Okubo T, Okuda S, Kimura H, Tsuchida A 2006 Colloid Polymer Sci. 284396

[3] Okubo T, Kanayama S, Ogawa H, Hibino M and Kimura K 2004 Colloid Polymer Sci. 282230

[4] Okubo T, Shinoda C, Kimura K, Tsuchida A 2005 Langmuir 219889

[5] Yamaguchi T, Kimura K, Tsuchida A, Okubo T and Matsumoto M 2005 Colloid Polymer Sci. 2831123
[6] Kimura K, Kanayama S, Tsuchida A, Okubo T 2005 Colloid Polymer Sci. $\mathbf{2 8 3} 898$

[7] Okubo T 2006 Colloid Polymer Sci. 2841191

[8] David J N, Erin G A, Linli M, Ruth H, Sciven L E 2004 Adv. Mater. 161393

[9] Okubo T, Kimura K, Kimura H 2002 Colloid Polymer Sci. 2801001

[10] Mclachlan M A, Johnson N P, Rue De la R M and Mccomb D W 2005 J. Mater. Chem. 15369

[11] Ye Y H, LeBlanc F, Hache A, Tuong V V2001 Appl. Phys. Lett. 7852

[12] Chung Y W, Leu I C, Lee J H and Hon M H 2004 Appl. Phys. A. 101007

[13] Routh A F, Russel W B, 1999 Langmuir 157762

[14] Leonid S, Amy Q S, Howard A S 2002 Langmuir 183441

[15] Deegan R D, Bakajin O, Dupout T F, Huber G, Nagel S R and Witeetn T A 1997 Nature 389827

[16] Palmer H J 1976 J. Fluid Mech. 75487

[17] Higuera F J 1987 Phys. Flulid 30679

[18] Prosperetti A, Plesset M S 1984 Phys. Fluids 271590

[19] Kennard E H 1938 Kinetic Theory of Gases (New York: McGraw-Hill) 